\title{
Diabetic Foot Ulcer (DFU) and Its Pharmaceutical and Therapeutic Strategies: A Systematic Review
}

\author{
Mahboobe Khodadadi ${ }^{1}$, Samaneh Naeimi ${ }^{2}$, Narjes Davoodi ${ }^{3}$ \\ ${ }^{1}$ MSc student of Nursing, Student Research Committee, Ahvaz Jundishapur University of Medical \\ Sciences, Ahvaz, Iran. \\ ${ }^{2}$ Nursing Student, Student Research Committee, Abadan Faculty of Medical Sciences, Abadan, Iran. \\ Email: samanehnaeimi1377@gmail.com \\ ${ }^{3}$ Student Research Committee, Dezful University of Medical Sciences, Dezful, Iran.
}

\begin{abstract}
Introduction: One of the complications of diabetes is diabetic foot ulcers which is very common in these patients. Given that in Studies, found that diabetic foot complications increase with age. Therefore, considering the importance of diabetic foot ulcer treatment in diabetic patients, this systematic review was conducted on Diabetic foot ulcer (DFU) and Its pharmaceutical and therapeutic strategies.
\end{abstract}

Materials and Methods: In this systematic review, this integrated overview study was conducted based on the Broome method. The present study is a systematic review article that was conducted in English and Persian by searching articles in search engines, scholar, PubMed, Springer's reputable scientific sites and databases. Using the articles published in the last 20 years, it was based on the most important treatments for diabetic foot ulcers. In the first stage, 39 papers were found. Of these, 11 articles related to the topic that was published in the last 20 years were reviewed.

Results: In this study, several methods for the treatment of diabetic foot ulcers have been investigated. In a study, in the treatment of chronic ulcers of grade 3 diabetes mellitus based on Wegener's classification, ultrasound waves of low frequency with standard care of the wound compared with standard care from scarring alone initially caused Accelerated recovery of chronic diabetic foot ulcers especially in the second and third months, while after 6 months of follow up, there was no significant difference in wound healing.

Conclusion: In this study, as stated, our goal is to examine diabetic foot ulcer and their therapeutic and therapeutic strategies, that the results of various studies show that there are several therapeutic and therapeutic methods for treating diabetic foot ulcers. It is recommended that with screening and blood glucose control, prevent ulcers that cause amputation of the foot.

Keywords: Diabetic Foot Ulcer, Diabetic Patients, Therapeutic Strategies, Pharmaceutical

\section{Introduction}

Diabetes is a dangerous and metabolic disease of the last century that is characterized by chronic hyperglycemia and can cause damage to various organs of the person. And because of the increasing number of people with the disease, it has become a major public health problem in the world (1-8). 
Currently, the prevalence of type 2 diabetes in Iran is reported to be $7.7 \%$, which is estimated to reach $8.6 \%$ by 2025 . A foot ulcer is a major complication of diabetes mellitus with high morbidity, mortality, and associated costs (9-11).

Foot ulcers are frequently infectious in diabetic patients and have the potential to progress to cellulite, and if not treated promptly and appropriately, can lead to blood and gangrene infections and sometimes lead to amputation. Preventive measures for diabetic foot, therefore, include identifying people with risk factors for foot ulcers, educating the patient and their companions about foot care, and appropriate and comprehensive treatment of foot ulcers (9), (12-16).

Chronic skin ulcers in diabetic patients usually occur in the lower extremities, especially the foot, which affects $15 \%$ of diabetic patients. A diabetic foot ulcer is the most common reason for hospitalization of diabetic patients $(17,18)$.

Diabetic foot ulcer has a profound effect on the quality of life of patients and imposes a huge burden on health care providers. Diabetic foot ulcers cause lower-extremity amputation in $85 \%$ of cases. Also, the mortality rate in diabetic foot ulcer patients compared to other diabetic patients is equal $(19,20)$.

Given that in the study by Al-Rubeaan et al (21) and Vilma et al (22) also found that diabetic foot complications increase with age. Therefore, considering the importance of diabetic foot ulcer treatment in diabetic patients, this systematic review was conducted on Diabetic foot ulcer (DFU) and It's pharmaceutical and therapeutic strategies.

\section{Materials and Methods}

The present study is a systematic review article that was conducted in English and Persian by searching articles in search engines, scholar, PubMed, Springer's reputable scientific sites and databases. Using the articles published in the last 20 years, it was based on diabetic foot ulcer (DFU) and pharmaceutical and therapeutic strategies. In the first stage, 39 papers were found. Of these, 11 articles related to the topic that was published in the last 20 years were reviewed.

In this systematic review, this integrated overview study was conducted based on the Broome method in order to achieve the goal of the study and to improve the study's thorough understanding and comprehension. This method is carried out in three stages of the search of texts, data evaluation, and data analysis so that in the search stage, the texts of post-retrospective studies are examined in four stages in terms of inclusion criteria. After obtaining the conditions for entry into the study, the content of the study is evaluated, and at the end of the analysis of the data.

The studies studied were written in English or Persian, access to their full text was possible, that entered the study, and unnamed and non-academic studies were deleted. To achieve relevant studies, a wide range of keywords including Diabetic Foot ulcer, Diabetic patients, therapeutic strategies and pharmaceutical was used as a one-to-one search, combined with the method "And" and "OR".

\section{Results}

Chronic skin ulcers in diabetic patients usually occur in the lower extremities, especially the foot, which affects $15 \%$ of diabetic patients. A diabetic foot ulcer is the most common reason for hospitalization of diabetic patients $(17,18)$.

In this review study, we intend to investigate diabetic foot ulcers and their therapeutic and therapeutic strategies by investigating 11 studies. 
In one study, the patient was advised to wash the wound thoroughly with a serum first, then place the mixture on a sterile gauze and place it on the wound and apply the dressing every 24 hours, then five days thereafter. After treatment, granule tissue developed in the wound and the wound healed completely within a month (23).

In another study, laser therapy was performed for 12 sessions over a 4-week low power laser, aluminum laser. Low-level laser radiation is non-contact with the lesion surface; changes in the lesion area were monitored and evaluated as the primary outcome after treatment and by the fourth month (24).

In a study, 35 patients with platelet gel and 35 subjects received routine treatment to determine the effect of platelet gel in the treatment of diabetic foot ulcers. There was no indication of amputation in the platelet gel treatment group. In the control group, foot ulcers in 6 patients resulted in limb amputation (25).

In another study, ultrasound waves of low frequency with standard care of the wound compared with standard care from scarring alone initially caused Accelerated recovery of chronic diabetic foot ulcers, especially in the second and third months, while after 6 months of follow up, there was no significant difference in wound healing (26).

Also, in a study that aimed to determine the effect of compressive-suction treatment (VCT) on foot ulcer healing in diabetic patients, when the treatment of Compressive suction with the appropriate care of the foot ulcer will increase the repair of the diabetic foot ulcer (27).

In another study, red light radiation around the wound and red intravenous light with an infrared laser to some acupuncture points for 5 to 10 days a day and then 2 times a week until complete recovery Wounds have been repairing these wounds (28).

Also, in a study, penicillin powder and rinse with betadine were stopped and treated as a daily dressing with topical mixture of heat-treated lambs in olive oil and animal oils, after a week after starting treatment, the new tissue was observed in the wound and the ulcer was completely closed within 2 weeks (29).

In another study, immersion ultrasound was debrided to necrotic tissue and the wound surface decreased and granulation tissue increased and wound healing improved without scar formation (30).

Other findings of the study showed that a period of cold endurance and plasma training had a significant effect on wound healing in diabetic rats. This is the first time that cold plasma stability and treatment have been used to improve diabetes Together, they significantly accelerate wound healing in diabetic rats (31).

Apligraft is another method that replaces the two-layer human skin that is used to treat intra venous ulcers and diabetic foot ulcers (32).

In a clinical trial, tretinoin $0.5 \%$ solution for 10 minutes daily followed by gel iodine for 4 weeks was effective in wound healing than the control group (33).

\section{Discussion}

Diabetic foot ulcer has a profound effect on the quality of life of patients and imposes a huge burden on health care providers. Diabetic foot ulcers cause lower-extremity amputation in $85 \%$ of cases. Also, mortality rate in diabetic foot ulcer patients compared to other diabetic patients is equal $(19,20)$. 
Given that in the study by Al-Rubeaan et al (21) and Vilma et al (22) also found that diabetic foot complications increase with age. Therefore, considering the importance of diabetic foot ulcer treatment in diabetic patients, this systematic review was conducted on Diabetic foot ulcer (DFU) and It's pharmaceutical and therapeutic strategies.

According to study, penicillin powder and rinse with betadine were stopped and treated as a daily dressing with a topical mixture of heat-treated lambs in olive oil and animal oils, after a week after starting treatment, the new tissue was observed in the wound and the ulcer was completely closed within 2 weeks (26).

The laser works on a number of mechanisms, firstly, the dilation of the vessels increases circulation in the area, helping to strengthen the immune system by destroying microorganisms in infectious wounds, and ultimately by strengthening the immune system. The process of healing the tissues strengthens the wound healing.

Another study aimed to determine the effect of compressive-suction treatment (VCT) on foot ulcer healing in diabetic patients, which according to the results suggests that patients with diabetic foot ulcers and chronic ulcers without improvement, for wound healing and limb restoration Use the most sophisticated pressuresaving treatment (27). Also in a similar study, a randomized clinical trial was performed to evaluate vacuum compression (VCT) to improve diabetic foot ulcers. Eighteen diabetic patients with foot ulcers were used by simple indirect sampling. Subjects were randomly divided into either experimental or control groups. Before and after the intervention, the level of foot ulcers was estimated serologically based on the Cavalieri principle. The experimental group was treated with VCT in addition to the usual treatment for 10 sessions. The control group received only conventional treatment including debridement, blood glucose control drugs, systemic antibiotics, wound healing with normal saline, discharge (pressure reduction), and daily wound dressing. After treatment, the experimental group significantly improved in measures of foot ulcer surface area compared with the control group. VCT enhances diabetic foot ulcer healing when combined with appropriate wound care (34).

\section{Conclusion}

In this study, as stated, our goal is to examine diabetic foot ulcer and their therapeutic and therapeutic strategies, that the results of various studies show that there are several therapeutic and therapeutic methods for treating diabetic foot ulcers. It is recommended that with screening and blood glucose control, prevent ulcers that cause amputation of the foot.

\section{Acknowledgment}

The authors of this article would like to thank everyone who has contributed to this article.

\section{References}

1. Khanpaye A, Abdoullahi A, Riahipour B, Madmoli Y, Mohebifar M, Mahmoodi R, Shirmardi S, Madmoli M. Evaluation of Knowledge, Attitude and Performance Regarding Gestational Diabetes Mellitus in Southwest of Iran. Journal of Pharmaceutical Research International. 2019 Jul 11:1-6.

2. Moslemirad M, Madmoli M, Madmoli Y, Niksefat M. Prevalence of type 1 and type 2 diabetes and its related factors in diabetic patients hospitalized in Khatam-ol-Anbia hospital in Shoushtar, 2014-15: A retrospective study. Journal of Research in Medical and Dental Science. 2018;6(3):421-6 
3. Feyzi R, Madmoli M. Epidemiologic approach to quality of life in diabetic patients: a systematic review study. IJMBS [Internet].2019;3(5): 24-28. Available from:

http://www.ijmbs.info/index.php/ijmbs/article/view/226

4. Madmoli M, Madmoli M, Aliabad MA, Khodadadi M, Ahmadi FP. A systematic review on the impact of empowerment in improving self-care behaviors and some other factors in diabetic patients. International Journal of Health and Biological Sciences. 2019 Apr 13;2(1):11-6.

5. Rostami F, Madmoli M, MirsamiYazdi N, Baraz Sh. Evaluation of The Prevalence of Lower Limb Amputation and Its Related Factors in Diabetic Patients Admitted to KHatam-ol-Anbia Hospital in Shoushtar During The 2015-2016: A Retrospective Study. International Journal of Ecosystems and Ecology Science (IJEES). 2018 June; 8 (3): 553-60.

6. Madmoli M, Izadi M, Khodadadi M, Khodadadi M, Papi Ahmadi F, Abbaszade Aliabad M.A Systematic Review Study on the Effect of Multimedia Training on Self-Care Behaviors and Quality of Life in Diabetic Patients. Journal of Genetics and Genetic Engineering, 2019; 2(4): 1-6.

7. Raisifar Z, Afshar Nia A, MaghamesiMoarrefi H, Madmoli M. Evaluation of Gi Bleeding Prevalence and Its Related Factors in Diabetic Patients Hospitalized in KHatam-ol-Anbia Hospital During 2015-16: A Retrospective Study. International Journal of Ecosystems and Ecology Science (IJEES). 2018 June; 8 (3): 609-14.

8. Madmoli M, Modheji Y, Rafi A, Feyzi R, Darabiyan P, AfsharNia A. Diabetes and its predictive role in the incidence of Alzheimer's disease. Medical Science, 2019; 23(95), 30-34

9. Madmoli M Rostami F, MirsamiYazdi N, Mosavi A, Baraz Sh. Evaluation of Prevalence of Diabetic Foot Ulcer and Its Related Factors in Diabetic Patients Admitted to KHatam-ol-Anbia Hospital in Shoushtar During 2015-2016: A Retrospective Study. International Journal of Ecosystems and Ecology Science (IJEES). 2018 June; 8 (3): 545-52.

10. Madmoli M, Madmoli Y, Taqvaeinasab H, Khodadadi M, Darabiyan P, Rafi A. Some influential factors on severity of diabetic foot ulcers and Predisposing of limb amputation: A 7-year study on diabetic patients. International Journal of Ayurvedic Medicine. 2019 Apr 3;10(1):75-81.

11. Madmoli M, Abbaszade Aliabad M, Madmoli M, Khodadadi M, Papi Ahmadi F. The Effect of Some Factors on Self-Care in Diabetic Patients: A Systematic Review. Journal of Genetics and Genetic Engineering. 2019; 3(1): 21-25

12. Madmoli M. A systematic Review Study on the Results of Empowerment-Based Interventions in Diabetic Patients. Int. Res. Med. Health Sci., 2019; 2(1):1-7.

13. Madmoli M, MahmoudiDehcheshmeh Z, Rafi A, Zahra Kord, FaribaMobarez, Pouriya Darabiyan. The rate of some complications and risk factors of diabetes in diabetic patients: Study on cases of 3218 diabetic patients. Medical Science, 2019; 23(95), 63-68

14. Madmoli M, Madmoli Y, Khodadadi M, Samsamipour M. Study of Some Effective Treatments for Accelerating Diabetic Foot Ulcer Healing: A Systematic Review. International Journal of Research Studies in Science, Engineering and Technology. 6(2), 2019; 34-39

15. Madmoli M, Madmoli Y, Khodadadi M, Samsamipour M. Factors Affecting the Level of Glycosylated Hemoglobin in Patients with Diabetes: A Systematic Review. Annals of Microbiology and Infectious Diseases 2(1), 2019; 43-47.

16. Madmoli M, Madmoli Y, Khodadadi M, Samsamipour M. Some Factors Affecting Quality of Life in Patients with Diabetes: A systematic Review. Annals of Microbiology and Infectious Diseases, 2(1), 2019; 26-30. 
17. Okonkwo UA, DiPietro LA. Diabetes and Wound Angiogenesis. Int J Mol Sci. 2017 Jul 3; 18(7): 1419. doi:10.3390/ijms18071419

18. Aallaa M, Sanjari M, Shahbazi S, Shayeganmehr Z, Abooeirad M, Amini MR et al. Diabetic foot workshop: Improving technical and educational skills for nurses. Med J Islam Repub Iran. 2017; 31.8. doi: $10.18869 /$ mjiri.31.8.

19. Schaper NC. Diabetic foot ulcer classification system for research purposes: a progress report on criteria forincluding patients in research studies. Diabetes MetabRes Rev 2004; 20 Suppl 1: S90-5.

20. Janmohammadi N, Moazzezi Z, Ghobadi P, Haddadi R, Montazeri M. Evaluation of the risk factors of diabetic foot ulcer and its treatment in diabetic patients, Babol, North of Iran. Iranian Journal of Endocrinology and Metabolism. 2010 Mar 15;11(2):121-5.

21. Al-Rubeaan K, Al Derwish M, Ouizi S, Youssef AM, Subhani SN, Ibrahim HM, et al. Diabetic foot complications and their risk factors from a large retrospective cohort study. PLoS One. 2015 May 6; 10(5): e 0124446. doi: 10.1371/journal.pone.0124446

22. UrbančičRovan V, Rovan J. An exploration of diabetic foot screening procedures data by a multiple correspondence analysis. Slovenian Journal of Public Health. 2017 Mar 1; 56(1):65-73. doi: 10.1515/sjph-2017-0009.

23. Zahmatkash M, Rashidi M. Treatment of Diabetic Leg Ulcer by Local Application of Honey and Olive Oil Mixture. JMP. 2009; 4 (29) :36-40. URL: http://jmp.ir/article-1-381-fa.html.

24. AtayiFeshtemi L, EsmaeiliJavidGh, Kaviani A, Larijani B, Razavi L, Salami M. Low Level Laser Therapy in Treatment of Diabetic Foot Ulcers: Double Blind Controlled Clinical Trial. Lasers in medicine. 1387; 5 (3).

25. Kargar S, JavadzadehShahshahani H, Tabkhi N. The effect of platelet gel on the treatment of diabetic foot ulcer. Sci J Iran Blood Transfus Organ. 2010; 6 (4) :283-291. URL: http://bloodjournal.ir/article-1-368-fa.html.

26. Amini S, RezaeiHemami M, Anabestani Z, Shayegan Mehran Z, Ardeshirralijani M B, Mohajeri Tehrani M R. The effect of low-frequency ultrasound waves on diabetic foot ulcers with osteomyelitis in a randomized clinical trial. 2011; 543-552.

27. Moodi H, Akbari A, Ghiasi F, Mahmoudzadeh H, Heidari Z, Rashidi H. The Effect of VacuumCompression Therapy (VCT) on the Diabetic Foot Ulcer Healing. zumsj. 2006; 14 (57) :15-22. URL: http://zums.ac.ir/journal/article-1-128-fa.html

28. N. Kazemi-Khoo. Successful treatment of diabetic foot ulcers with low-level laser therapy. The foot, December 2006; 16(4): 184-187.

29. Khademhh, koushan y, asgharzadehA. Treatment of diabetic foot ulcer with propolis and olive oil: a case report. 2012; 35-38.

30. JaimeeHaan PT, Sharon Lucich PT. A Retrospective Analysis of Acoustic Pressure Wound Therapy: Effects on the Healing Progression of Chronic Wounds. J Am Col Certif Wound Spec 2009; 1(1): 28-34.

31. Aryandoost S, Nazarali P, Fathi R, Sohbatzadeh F. The effect of endurance exercise on wound healing of diabetic rats by cold plasma. Iranian Journal of Diabetes and Metabolism. 2017; 16 (2) :95-102. URL: http://ijdld.tums.ac.ir/article-1-5495-fa.html

32. RonaldsWarren.http://www.fda.gov/MedicalDevices/ProductsandMedicalProcedures/Device ApprovalsandClearances/Recently- ApprovedDevices/ucm085085.htm 
33. McCulloch D, Asla R. Management of diabetic foot lesions. Up to Date Ver 18.1 (updated 31 Jan 2010).

34. Akbari A, Moodi H, Ghiasi F, Sagheb HM, Rashidi H. Effects of vacuum-compression therapy on healing of diabetic foot ulcers: Randomized controlled trial. Journal of Rehabilitation Research \& Development. 2007 Sep 1; 44(5).

How to cite this Article: Mahboobe Khodadadi ${ }^{1}$, Samaneh Naeimi2*, Narjes Davoodi ${ }^{3}$; Diabetic Foot Ulcer (DFU) and Its Pharmaceutical and Therapeutic Strategies: A Systematic Review

Int. J. Drug Res. Dental Sci., 2019; 1(2):19-25.

Source of Support: Nil, Conflict of Interest: None declared. 